\title{
OPINIONES
}

\section{REDD+ en Latinoamérica: el caso de Perú}

\author{
REDD+ in Latin America: the case of Peru
}

\author{
Brenton Ladd a,b*, Pablo L Peri ${ }^{\mathrm{c}}$ \\ *Autor de correspondencia: ${ }^{\text {a } U n i v e r s i t y ~ o f ~ N e w ~ S o u t h ~ W a l e s, ~ E v o l u t i o n ~ a n d ~ E c o l o g y ~ R e s e a r c h ~ C e n t r e, ~ S c h o o l ~ o f ~ B i o l o g i c a l, ~}$ \\ Earth and Environmental Sciences, Sydney, NSW 2052, Australia, brenton.ladd@unsw.edu.au

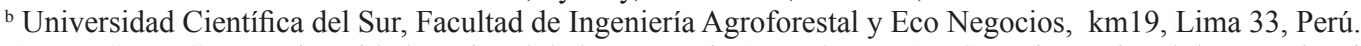 \\ c INTA EEA Santa Cruz, Universidad Nacional de la Patagonia Austral (UNPA), Consejo Nacional de Investigaciones \\ Científicas y Técnicas (CONICET)-cc332 (9400) Rio Gallegos, Santa Cruz, Argentina, pperi@correo.inta.gov.ar
}

\begin{abstract}
SUMMARY
The question of how developing countries like Peru can participate effectively in the REDD+ (reducing emissions from deforestation and forest degradation) policy initiative of the United Nations has been the focus of considerable debate recently. The aim of this study was to detect the main challenges associated with monitoring deforestation and forest degradation and also to analyze the policy challenges associated with implementing REDD in Peru. To participate effectively in this process, it is essential that the Peruvian national government formulates a national response since it is unlikely that provincial agencies managing natural resources will be able to develop the necessary expertise to carry out effective monitoring, reporting and verification, and because the underlying factors driving deforestation will require a large investment and a coordinated response at the country level.
\end{abstract}

Key words: REDD, carbon, deforestation, forest degradation, carbon dioxide.

\section{RESUMEN}

La cuestión de cómo los países en vías de desarrollo como el Perú pueden participar efectivamente en el marco REDD+ (reducción de emisiones de la deforestación y degradación de bosques) de las Naciones Unidas ha sido el foco de considerables debates recientemente. El objetivo del presente trabajo fue detectar los principales aspectos de monitoreo de degradación forestal y decisiones políticas para enfrentar un proceso REDD en Perú. Para participar de manera efectiva en este proceso, es esencial que el gobierno nacional peruano formule una respuesta nacional ya que es poco probable que las entidades provinciales de manejo de recursos naturales puedan desarrollar la experiencia necesaria para efectuar el monitoreo eficaz y porque los factores subyacentes de la deforestación requieren de una gran inversión y una amplia visión a nivel país.

Palabras clave: REDD, carbono, deforestación, degradación de bosques, dióxido de carbono.

\section{INTRODUCCIÓN}

REDD (reducción de emisiones de la deforestación y degradación de bosques) es un marco político que está siendo desarrollado por las Naciones Unidas (ONU) y que tiene como objetivo aprovechar los pagos de los mercados emergentes de carbono para limitar la deforestación y la degradación de los bosques en el mundo en desarrollo (UNFCCC 2007). Este es un mecanismo prometedor a través del cual los países industrializados podrían compensar sus emisiones de dióxido de carbono mediante la inversión en conservación en el mundo en vía de desarrollo. Dado que la mayor parte de la deforestación y degradación de los bosques sucede en los países en desarrollo, la creación de la esquema REDD como un sistema internacional de compensación dirigido a los dueños de las áreas forestales en esos países constituye una importante iniciativa para promover un manejo forestal sustentable a escala global que logre conservar la cubierta forestal para reducir emisiones de $\mathrm{CO}_{2}$ (Strassburg et al. 2010). Hay propuestas que promueven la definición de un mecanismo REDD+ bajo una visión integral que incluya la disminución de emisiones, la conservación de las reservas de carbón, la conservación de la biodiversidad, la disminución del deterioro así como el fortalecimiento del capital social y la mejora de la vida rural, a través de una serie de acciones dirigidas al manejo sustentable de los bosques (Miles y Kapos 2008). Por lo tanto, para la aplicación del mecanismo REDD+ es fundamental socializar esta discusión en cada país y avanzar hacia la construcción de una estrategia participativa para REDD, que refleje las propuestas y visiones de los diferentes actores involucrados en los bosques (Hajek et al. 2011). La idea es que las poblaciones vinculadas a los bosques reciban apoyo técnico y financiero internacional para 
conservar sus bosques y para realizar su aprovechamiento sostenible, utilización de la madera y productos forestales no maderables, sin sobrepasar su capacidad de regeneración natural. De esta manera los usuarios y habitantes del bosque podrían mejorar sus condiciones de vida, generando una nueva economía sostenible del bosque y garantizando el mantenimiento en pie de los bosques nativos. Sin embargo, para recibir este apoyo del gobierno nacional peruano, primero debe comprometerse con el programa REDD de la ONU, con la elaboración de un "plan nacional de REDD" que todavía está en el etapa de evaluación (REDD 2012). En este contexto, el objetivo del presente trabajo fue detectar los principales aspectos de monitoreo de degradación forestal y decisiones políticas para enfrentar un proceso REDD en Perú.

\section{MONITOREO}

REDD+ requiere de un sistema transparente y sólido de monitoreo ya que va a determinar el precio del carbono cuando los mercados comiencen a operar (Venter y Koh 2012). En la actualidad sólo el 3 \% de los países tiene una capacidad de monitoreo adecuada (Venter y Koh 2012). La deforestación y la degradación forestal en los trópicos es a menudo el resultado del desmonte a pequeña escala por los agricultores de subsistencia, por lo que es necesario un sistema de monitoreo con una escala espacial de menos de una hectárea si se trata de detectar con precisión la degradación forestal (Ryan et al. 2012). En este sentido Gilbert (2012) resaltó que el potencial para estimar el área deforestada y degradada de la India utilizando imágenes satelitales de MODIS/LANDSAT es limitado, indicando la necesidad de nuevas tecnologías con alta resolución. Actualmente se cuenta con dos tecnologías emergentes que pueden cumplir los requisitos de REDD+ para la transparencia y solidez del proceso. La primera, denominada Airborne light detection and ranging (LIDAR), es una tecnología de detección remota que utiliza pulsos de láser para medir distancias entre los objetos (el suelo y las copas de los árboles) (Asner et al. 2012). LIDAR, en combinación con mediciones de acumulación carbono realizadas en campo y el uso de ecuaciones alométricas, ha demostrado ser una herramienta de medición efectiva (Asner et al. 2010). La generación de datos LIDAR está, sin embargo, más allá del alcance de las entidades de manejo de recursos naturales provinciales, porque se requiere que los sensores del LIDAR se acoplen a una avioneta y que las áreas a ser evaluadas sea sometidas a sobre vuelos. Esto es obviamente costoso y, por lo tanto, fuera del alcance de la mayoría de las entidades provinciales de manejo de recursos naturales. En segundo lugar, los datos LIDAR resultantes deben ser procesados por un técnico con una formación altamente especializada que no se pueden encontrar fácilmente en las entidades provinciales de manejo de recursos naturales. Un proyecto en el sureste de Perú ha demostrado que esta tecnología puede producir mapas de carbono del bosque amazónico de alta calidad (Asner et al. 2010). Por otro lado, no es claro que los sobre vuelos sean efectivos para el mapeo de la acumulación de carbono en las regiones montañosas del Perú, donde la nubosidad persistente limita el uso de aviones ligeros y LIDAR.

Una segunda tecnología emergente que podría cumplir los requisitos de REDD+ es la tecnología de Radar Remote Sensing (RRS) que utiliza imágenes satélites (Agrawal et al. 2011, Ryan et al. 2012, SIC 2012). RRS es menos preciso que LIDAR, pero tiene la ventaja de que se cuenta actualmente con la cobertura completa de imágenes de los trópicos y que la calidad de dichas imágenes no se ve afectada por la nubosidad persistente. Esto podría ser una ventaja significativa en un país como el Perú, donde la nubosidad y el terreno montañoso pueden limitar la cobertura potencial de LIDAR. Sin embargo, como en el caso de LIDAR, se requiere de técnicos altamente especializados con la capacidad de manipular los datos de RRS.

En ambos casos (LIDAR y RRS), la formación de personal especializado será fundamental para que las organizaciones puedan efectuar el monitoreo. El desarrollo de esta capacidad de monitoreo requerirá de una importante inversión en la educación, que puede ser demasiado costosa para las entidades provinciales de manejo de recursos naturales. El gobierno peruano podría seguir el modelo argentino y chileno de financiamiento de los estudiantes de doctorado para que se capaciten en el extranjero (EE.UU. para LIDAR y Japón, EE.UU. o Inglaterra para RSS) para adquirir los conocimientos necesarios y con el requisito de que cuando terminen, regresen al Perú para ayudar en la gestión de los recursos naturales y la formación de la próxima generación de gerentes de recursos naturales (los analistas espaciales en este caso) en el Perú.

Finalmente, en el campo peruano (la Amazonía y la Sierra) hay personas extremadamente pobres. A menudo se vive sin desagüe, electricidad y agua potable (Zwane 2007). La capacidad de los gobiernos provinciales de recaudar impuestos es limitada. Con la limitada cantidad de dinero que los gobiernos provinciales tienen a su disposición no parece razonable sugerir que se debe invertir en el desarrollo de la capacidad de monitoreo, reporte y verificación (MRV) necesario por REDD cuando el pueblo de la región no tiene la infraestructura básica. Además, la capacidad de hablar y escribir en inglés es limitada en la comunidad rural, pero no tanto en la capital Lima. Con la capacidad de hablar y escribir inglés existe la posibilidad de presentar las solicitudes de subvención a los organismos de financiación internacionales.

\section{LA DESCENTRALIZACIÓN Y LA FORMULACIÓN DE POLÍTICAS}

La cuestión de cómo los países en vías de desarrollo como el Perú pueden participar más efectivamente en este proceso ha sido el foco de considerable debate recientemente. En el Perú hay un consenso creciente acerca de la 
necesidad de un movimiento hacia la descentralización de la gestión de los recursos naturales en general, y de manera más específica en la implementación de REDD+ (CastroNúñez et al. 2010). El debate sobre la potencial efectividad de la descentralización en las respuestas políticas en América Latina no está de ninguna manera restringida al Perú (Toni 2011). Pero en Perú se argumenta que la descentralización es la mejor estrategia posible debido a la diversidad cultural y ecológica en dicho país (Castro-Núñez et al. 2010). Pero, en un mundo globalizado, la descentralización no significa que el proceso sea democrático, y en su lugar podría producirse una balcanización donde se reduce la capacidad para una formulación de políticas efectivas. En el caso de Perú se sostiene que la descentralización es un error en el contexto de REDD+, y que su éxito sólo será posible si se aborda a nivel nacional. El emergente marco en REDD+ para el monitoreo de la deforestación y la degradación forestal es técnicamente complejo y requerirá del desarrollo de técnicas avanzadas en teledetección y el uso de sistemas de información geográfica. Si pequeñas cantidades de dinero se invierten en muchas entidades para la gestión de los recursos naturales, para fomentar la capacidad de monitoreo en las provincias, es probable que la inversión no tenga el impacto deseado. Esto es debido a que las capacidades desarrolladas probablemente estén muy por debajo de las avanzadas capacidades de análisis espacial que se necesitan para REDD+. Sin embargo, si los recursos fueran invertidos en un instituto central, dedicado al análisis espacial, es más probable que el Perú pudiese desarrollar la capacidad para cumplir con los requisitos complejos, rigurosos, creíbles y emergentes en el marco de REDD+.

Otra importante razón para la formulación de estrategias de REDD+ en políticas a nivel nacional es la necesidad de atender las causas de la deforestación (UNFCCC 2007). Un proceso descentralizado del manejo de recursos naturales es poco probable que conduzca a una respuesta política viable a la REDD+ en donde se identifiquen y alivien las causas de la deforestación y degradación forestal, y se logre la estabilización de las reservas de carbono de los bosques. En el Perú las causas subyacentes de la deforestación y la degradación de los bosques en la cuenca amazónica se deben principalmente a la migración de los campesinos de Los Andes a la Amazonía (Naughton-Treves 2004, Zwane 2007, Castro-Núñez et al. 2010, Velarde et al. 2010). Cualquier intento de limitar la deforestación tropical en este país tendrá que invertir en una estrategia que efectivamente contempla este aspecto social a nivel supra-regional.

Por ende, se necesita de inversiones que apunten a mejorar la vida de los habitantes de Los Andes. La región andina (altiplano) fue la sede de una antigua civilización avanzada (el Imperio Inca) y por ello es claro que esta región puede ofrecer las necesidades vitales a sus habitantes. Sin embargo, el hecho de que las personas estén abandonando la región es un evidente indicio de que las necesida- des de la población no se están cumpliendo. Las razones de esto son complejas, pero es notable el hecho de que el marco institucional en el Perú haya favorecido la búsqueda de rentas (la extracción de recursos) por sobre la inversión que tienda a aumentar la capacidad productiva (Reid 2007, Brack-Egg y Mendiola-Vargas 2010). En el mediano plazo esta dinámica podría minimizarse a través de la revegetación en el altiplano con la financiación conjunta del mecanismo de desarrollo limpio (CDM) de las Naciones Unidas y el World Bank Carbon Partnership Facility. Sin embargo, la reconstrucción de los ecosistemas naturales originales degradados por acción antrópica por milenios en el Altiplano es poco viable. En lugar de restauración, el esfuerzo podría estar centrado en la tarea más simple de la construcción de los ecosistemas que satisfagan las necesidades humanas. La revegetación de las zonas de captación de agua en la región, por ejemplo, podría asegurar que, cuando el cambio climático se intensifique y los glaciares se derritan, las cuencas puedan continuar proporcionando una fuente limpia y confiable de agua potable en la región. Esto a su vez mitiga la necesidad de emigrar de la región en el futuro. También la plantación de árboles en cortinas cortavientos podría aumentar la captura de carbono y, por lo tanto, es una actividad que podría realizarse en el marco del mecanismo de desarrollo limpio. Ello mejoraría la calidad de vida de las personas que viven en la región a través de: 1) la mejora de la productividad en la cría de ganado (una importante actividad económica en la región (BrackEgg y Mendiola-Vargas 2010) y 2) proporcionar una fuente de combustible, ya que la mayoría de los habitantes de la región sigue siendo muy dependiente de la leña como fuente primaria de combustible. Por último, el mecanismo de desarrollo limpio se podría utilizar para cofinanciar la construcción de cocinas con un sistema de aislamiento que sean más eficientes. Esto podría reducir la demanda de madera y, por lo tanto, reducir las emisiones de carbono, al mismo tiempo que mejoraría la calidad de vida de las personas en la región mediante la reducción del volumen de leña que necesitan recoger. El hecho de que las cocinas eficientes estén aisladas también significa que los hogares que las utilizan, estén libres de humo, lo que reduciría la incidencia de enfermedades respiratorias. Sin embargo, es importante resaltar que existen limitantes de tipo financieros (costos transaccionales y precio de los certificados de emisiones reducidas, CER) y sociales (por ejemplo, la gobernanza de los proyectos y forma que los beneficios económicos llegan a la gente), entre otros aspectos, para el éxito de las estrategias planteadas en el marco de REDD+.

\section{CONCLUSIONES}

REDD+ y el mecanismo de desarrollo limpio podrían ser instrumentos útiles para el fortalecimiento de las políticas de conservación en el Perú. El problema de deforestación no es insalvable. Hay numerosos ejemplos de cómo se podría disminuir la deforestación como la certificación 
forestal (FSC-Perú 2012), el fomento de actividades de impacto mínimo, el ecoturismo y producciones alternativas como la producción de castaña y caucho (Hajek et al. 2011) o, como se ha demostrado en Brasil, el aumento del tamaño de las áreas protegidas (Nepstad et al. 2009). Para participar de manera efectiva y comprometerse con este proceso, es esencial que el Perú formule una respuesta nacional, porque: 1) es poco probable que las entidades provinciales de manejo de recursos naturales puedan desarrollar la experiencia necesaria para monitorear eficazmente y 2) es necesario abarcar los factores subyacentes de la deforestación que requieren de una gran inversión y una amplia visión a nivel país.

\section{AGRADECIMIENTOS}

Gracias a Pina Gervassi, Tatiana Lapeyre-Zárate, y los revisores anónimos por comentarios útiles sobre el borrador.

\section{REFERENCIAS}

Agrawal A, D Nepstad, A Chhatre. 2011. Reducing Emissions from Deforestation and Forest Degradation. Annual Review of Environment and Resources 36: 373-396.

Asner G, J Mascaro, H Muller-Landau, G Vieilledent, R Vaudry, M Rasamoelina, J Hall, M van Breugel. 2012. A universal airborne LiDAR approach for tropical forest carbon mapping. Oecologia 168: 1147-1160.

Asner GP, GVN Powell, J Mascaro, DE Knapp, JK Clark, J Jacobson, T Kennedy-Bowdoin, A Balaji, G Paez-Acosta, E Victoria, L Secada, M Valqui, RF Hughes. 2010. High-resolution forest carbon stocks and emissions in the Amazon. Proceedings of the National Academy of Sciences 107(38): 16738-16742.

Brack-Egg A, C Mendiola-Vargas. 2010. Ecología del Perú. $3^{\text {a }}$ edición. Lima, Perú. Bruno. 495 p.

Castro-Núñez A, E Gómez-Rivero, ED Lopez-Hurtado, M Allende, L Pedroni, R Hofstede. 2010. Plantilla de propuesta para la fase de preparación para REDD+ (Readiness Plan Proposal - R-PP). Lima, Perú. Ministerio del Ambiente del Perú. $228 \mathrm{p}$.

FSC-Perú (Forest Stewardship Council, PE). 2012. FSC Certificate Database. Disponible en http://info.fsc.org/

Gilbert N. 2012. India's forest area in doubt. Reliance on satellite data blamed for over-optimistic estimates of forest cover. Nature 489: 14-15.

Hajek F, MJ Ventresca, J Scriven, A Castro. 2011. Regime-building for REDD+: Evidence from a cluster of local initiatives in south-eastern Peru. Environmental Science \& Policy 14(2): 201-215.
Miles L, V Kapos. 2008. Reducing greenhouse gas emissions from deforestation and forest degradation: global land-use implications. Science 320(5882): 1454-1455.

Naughton-Treves L. 2004. Deforestation and carbon emissions at tropical frontiers: A case study from the Peruvian Amazon. World Development 32: 173-190.

Nepstad D, BS Soares-Filho, F Merry, A Lima, P Moutinho, J Carter, M Bowman, A Cattaneo, H Rodrigues, S Schwartzman. 2009. The end of deforestation in the Brazilian Amazon. Science 326(5958): 1350-1351.

REDD (Reducing Emissions from Deforestation and Forest Degradation). 2012. A database of REDD activities on the ground. Disponible en www.theredddesk.org/countries/ peru/activities)

Reid M. 2007. Forgotten continent: the battle for Latin America's soul. New Haven, US. Yale University Press. 400 p.

Ryan CM, T Hill, E Woollen, C Ghee, E Mitchard, G Cassells, J Grace, IH Woodhouse, M Williams. 2012. Quantifying small-scale deforestation and forest degradation in African woodlands using radar imagery. Global Change Biology 18(1): 243-257.

SIC (Satellite Imaging Corporation, US). 2012. Quickbird satellite sensor. Disponible en www.satimagingcorp.com/ satellite-sensors/quickbird.html.

Strassburg BBN, A Kelly, A Balmford, RG Davies, HK Gibbs, A Lovett, L Miles, CDL Orme, J Price, RK Turner, ASL Rodrigues. 2010. Global congruence of carbon storage and biodiversity in terrestrial ecosystems. Conservation Letters 3(2): 98-105.

Toni F. 2011. Descentralización y REDD+ en Brasil. In Petkova E, A Larson, P Pacheco eds. Gobernanza forestal y REDD+: Desafíos para las políticas y mercados en América Latina. Bogor, Indonesia. CIFOR. p. 61-77.

UNFCCC (United Nations Framework Convention on Climate Change, US). 2007. Reducing emissions from deforestation in developing countries: approaches to stimulate action, conference of the parties, Bali, 3 December to 11 December 2007. Available from http://unfccc.int/resource/docs/2007/ sbsta/eng/123a01r01.pdf. United Nations Framework Convention on Climate Change (UNFCCC). Bonn, Germany.

Velarde SJ, J Ugarte-Guerra, MR Tito, JL Capella, M Sandoval, G Hyman, A Castro, JA Marín, E Barona. 2010. Reducing Emissions from All Land Uses in Peru. Final National Report. Nairobi, Kenya. ASB Partnership for the Tropical Forest Margins. $142 \mathrm{p}$.

Venter O, LP Koh. 2012. Reducing emissions from deforestation and forest degradation (REDD+): game changer or just another quick fix? Annals of the New York Academy of Sciences 1249(1): 137-150.

Zwane AP. 2007. Does poverty constrain deforestation? Econometric evidence from Peru. Journal of Development Economics 84(1): 330-349. 